\title{
Anti-Collision Warning Algorithm based on Visual Perception in Front of Vehicle
}

\author{
Pengcheng Ding ${ }^{1}$, Jindong Zhang ${ }^{1,2, *}$, Yanghao Zhou ${ }^{1}$, Hongliang Liu ${ }^{1}$, \\ Xiao Wang ${ }^{1}$ \\ ${ }^{1}$ College of Computer Science and Technology, Jilin University, Changchun, 130012, China \\ ${ }^{2}$ Key Laboratory of Symbol Computation and Knowledge Engineering of the Ministry of Education, \\ Jilin University, Changchun,130012, China
}

*Corresponding author: Jindong Zhang (Email address: zhangjindong_100@163.com).

\section{Keywords: Computer Vision, Pattern Recognition, Rear-End Collision Warning.}

\begin{abstract}
The vehicles' driving safety plays an important role in the transportation safety's development, and it's a necessary requirement in intelligent transportation and intelligent vehicle. The point that is suggested in this paper is that vehicles' collision warning system which is based on computer vision, and the system is built on computer vision, pattern recognition, machine learning and some other artificial intelligent theory and techniques, discerning the vehicles which is in front of your vehicle and measure the security range, warning the possible danger timely to make sure your drive safe. We use the characteristic which is called haar of the samples to train in the classifier to get a cascaded classifier named Boosted, loading the classifier and image of the vehicles marked and calculating the distance and relative speed. In the last, we do a lot of system simulation experiments, verifying the accuracy and the effectiveness of the system from vehicle outline detection results and safe vehicle determination.
\end{abstract}

\section{Introduction}

In recent years, the road safety situation is getting grim. Road traffic accidents become a major nuisance in modern society, associating with advanced security technology research has received increasing attention [1]. If we can warn drivers before the accident and take some security measures, we will reduce the occurrence of probability traffic accidents. Rear-end collision warning system is based on reducing rear-end accident, implementation provides security information to the driver in driving process technology and equipment. Design of rear-end collision warning system based on image identification studies first vehicle video image recognition of vehicles on the highway, when the vehicle of a range alarm to reduce traffic accidents causing casualties and property losses [2].

The rear-end collision warning system is technological equipment based on reducing rear-ending accident, providing security information to the driver in driving process. So designing of rear-end collision warning system studies which are based on image identification can recognize the vehicles on the highway by vehicle video image recognition, and when the vehicle is identified, system can alarm the dangerous distance to reduce traffic accidents causing casualties and property losses.

\section{General description of the system}

Training the sample Harr features with classifier to get a cascade of boosted classifier. Samples trained are divided into positive samples and counter examples. Positive samples are the objective samples waiting for inspection, and counter examples samples mean any other pictures, all the sample pictures have been normalized to the same size. When the classifier training finished, they can be applied to detecting the regions of interest in the input image. The program flowchart is shown in Fig.1. The function called Haar Detect Objects in OpenCV functions, after loading the trained classifier, can return a series of rectangular sequence with targets, via OpenCV functions to manipulate the image to identify the vehicle. 


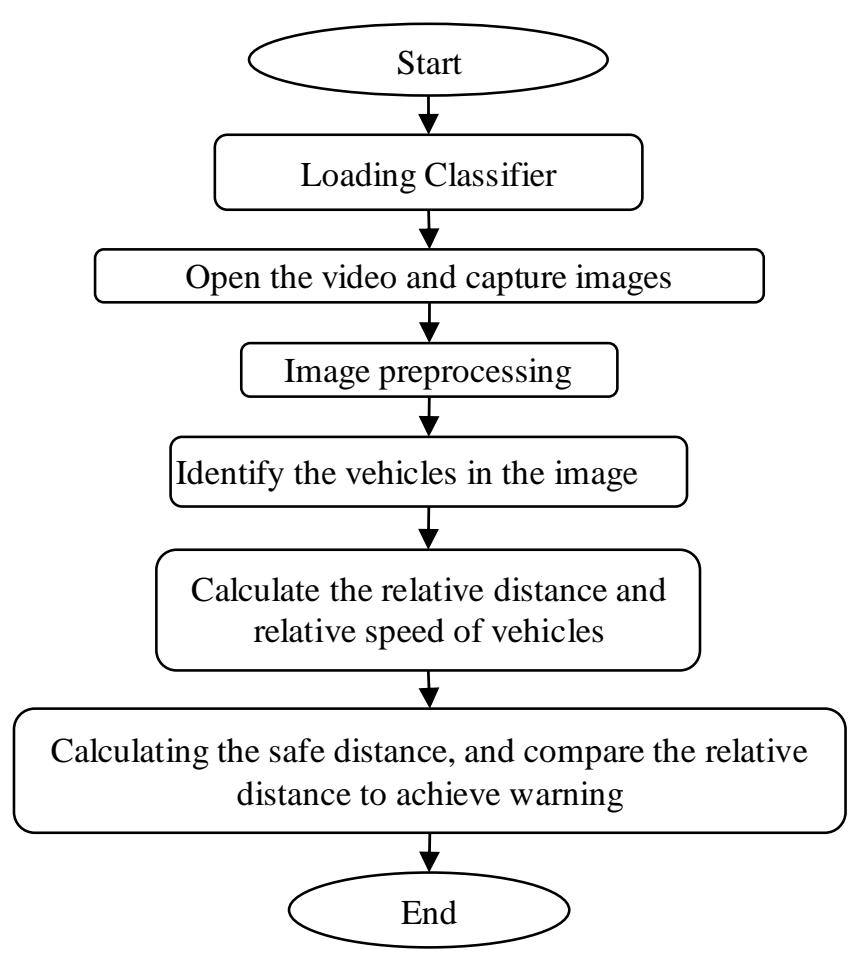

Fig. 1. The program flowchart

As program flowchart shows, when program runs, firstly it loads the trained classifier, and then pretreats the frame on the video. Through trained image to recognize the vehicles in the frame, and calculate the recognized vehicles' distance and speed in security distance formula, when the two vehicle's distance is less than security distance, system judges that it exists possible collision, and warn the driver, making sure the security of driving process.

\section{Vehicle identification}

The whole process of vehicle identification is as follows:

Step1.Calculate integral graph, integral of square graph and so on;

Step2. Testing: Calculating "for the current proportion", according to "the current step size", the number of movement in advance, so the detection window location can also be very convenient to be calculated. Under the current proportion, the classifier is based on detection of hidden Windows in different locations to get different hidden classifiers, actually at different window positions which is in the classifier and in the current ratio. We will calculate the address in integral graph, the integral of square graph. And later we can get values according to the offset value, and do some preparation. Then calculate the moved location.

Step3. We can find the rectangular area containing the target object, and return these areas as a series of rectangular box.

Step4. For each rectangle, vehicle i, we use the rectangle to draw in images.

For each frame, before call the cvHaarDetectObjects function, we should calculate integral graph, integral of square graph, as well as current ratio and the current stride length, and return the rectangle which contains the target object.

The AdaBoost algorithm is a simple weak classification algorithm which is still developing, through continuously training, it can improve the ability to classify data [3] [4]. The process of AdaBoost algorithm is as follows:

Step 1. Through learning the nth trained samples to get the first weak classifiers;

Step 2. Put the samples which are classified incorrectly and other new data to constitute a new nth training samples, through the study of this sample, we can get the second weak classifier;

Step 3. Put the wrong samples in Step 1 and Step 2 together with other new samples constituted another new nth training samples, basing on this sample study, to get a third weak classifier; 
Step 4. Finally, after that we get a strong classifier. That a data belongs to a kind of class is to be decided by all classifier's weights.

For every set of training set, by changing each of samples, we can get a different training set called $\mathrm{Si}$. For each $\mathrm{Si}$, we can train them to get a weak classifier called $\mathrm{Hi}$, and then we combine these classifiers based on different weights to get a strong classifier. For the first time, each sample obeys uniform distribution, through the train, and we can get a classifier called $\mathrm{H} 0$. In this training set, to the correct classification, we will reduce its distribution probability; to the wrong classification, we can improve its distribution probability, so the new training set S1 is mainly aimed at those which are the samples in not very good classifications. Then we use S1 trains, getting classifier H1 followed by an iteration assuming set iterations in addition to $T$, and getting $T$ th classifier. For each classifier's weights, the higher classification accuracy, the higher weights [5]. So based on Adaboost algorithm, we can use haar features of vehicles to train a classifier and use the classifier to identify vehicles in the photo.

\subsection{Distance Calculation Based on Monocular Vision}

We use camera to capture picture, projecting 3D scenes onto the 2D plane of camera. Pinhole camera model could basically meet the requirements of measuring objects in the Earth coordinate system. P1 is any point, and P2 is the projection point of P1. Point P2 on picture plane is the intersection point of picture plane and OC (Optical Center)-P1 line [6]. Camera images are converted into digital image and stored in $(\mathrm{u}, \mathrm{v})$ form which is in the Cartesian coordinate system. And establish a physical coordinate system in the image Center ( $\mathrm{u} 0, \mathrm{v} 0)$. Through this analysis, we know that: On the premise of camera calibration, If we have knew a point $\mathrm{P} 1$ 's world coordinates ( $\mathrm{Xw}, \mathrm{Yw}, \mathrm{Zw})$, we could use the formula to figure out the image coordinates $(\mathrm{u}, \mathrm{v})$ of the point.

\subsection{Principle of measuring object's distance}

Assuming world coordinate system is located at the location and dividing the target into $\mathrm{N}$ parts along the Yw direction. Each part is the approximation of a rectangle. Suppose that the 4 vertices of the $\mathrm{i}$-th rectangle are labeled as $\mathrm{P}_{1}^{\mathrm{i}}, \mathrm{P}_{2}^{\mathrm{i}}, \mathrm{P}_{1}^{\mathrm{i}+1}, \mathrm{P}_{2}^{\mathrm{i}+1}$. For imaging objects, measuring the distance between the camera and the target is actually measuring the distance between the camera and the point on the front surface of the target. For parts that cannot be imaged, it's not measurable. When choosing a point, feature point should be selected and using the distance from the camera optical center to feature point to measure the distance from object to camera. To measure the distance, the key is to find the representative feature points. In this paper, using the centroid of target as the feature point, and using image processing to calculate feature point, to get the full distance of feature points to the camera.

\subsection{Establishment of vehicle safety distance model}

The Model building thought which Mazda developed in the CW / CA system is that: The sensor mounted on the vehicle-following is found that the preceding vehicle is decelerating, It Starts sending information to the Safety distance alarm system. When the distance between the front vehicle and the following vehicle is less than the braking distance of vehicle-following, the distance between two vehicles is 5 meters the system delay time and the vehicle deceleration time in the model are measured by a large number of tests. In addition, the braking deceleration of the front vehicle and rear two vehicles is set according to the road condition [7]. The braking deceleration of the two automobiles in the model takes different values, It gives full consideration to such adverse circumstances that the deceleration performance of the following vehicle is not as good as that of the preceding vehicle, the formula is as follow:

$$
\text { dsafe }=\frac{1}{2}\left(\frac{v_{\text {follow }}^{2}}{a_{1}}-\frac{\left(v_{1}-v_{\text {relative }}\right)^{2}}{a_{2}}\right)+{ }_{\text {vfollow }} t_{1}+v_{\text {relative }} t_{2}+d_{0}
$$

where $\mathrm{v}_{\text {follow }}$ is the velocity of following vehicle, unit: $\mathrm{m} / \mathrm{s} ; \mathrm{v}_{\text {relative }}$ is the relative velocity of two vehicles, unit: $\mathrm{m} / \mathrm{s} ; \mathrm{a}_{1}$ is the braking speed of following vehicle, $\mathrm{a}_{1}=6 \mathrm{~m} / \mathrm{s}^{2} ; \mathrm{a}_{2}$ is the braking speed of front vehicle, $\mathrm{a}_{2}=8 \mathrm{~m} / \mathrm{s}^{2} ; \mathrm{t}_{1}$ is the deceleration time, $\mathrm{t}_{1}=0.1 \mathrm{~s} ; \mathrm{t}_{2}$ is the system delay time, $\mathrm{t}_{2}=0.6 \mathrm{~s}$; $\mathrm{d}_{0}$ is the distance of two vehicles after parking, $\mathrm{d}_{0}=5 \mathrm{~m} ; \mathrm{d}_{\mathrm{s}}$ is the safe distance, unit: $m$. The abovementioned model calculates the minimum safety distance. In practical application, the system will send an alarm when the distance between the two vehicles is less than this distance. Since during 
normal driving, the speed of each vehicle is close to uniform speed, and the speed of two vehicles is similar. So when the front vehicle velocity and the rear vehicle velocity calculated by this model are equal. The vehicle safety distance under common vehicle velocity is shown in Table 1:

Table 1.The vehicle safety distance under common vehicle velocity

\begin{tabular}{|c|c|c|c|c|}
\hline $\begin{array}{ll}\text { The speed of following vehicle } \\
\text { Relative speed }\end{array}$ & 10 & 20 & 30 & 40 \\
\hline 0 & 8 & 15 & 26 & 42 \\
\hline 10 & 20 & 40 & 64 & 92 \\
\hline 20 & 20 & 52 & 88 & 129 \\
\hline 30 & 52 & 101 & 154 & 211 \\
\hline
\end{tabular}

\section{Experiment and result analysis}

The algorithm is simulated on the Lenovo G510 PC. The experimental environment is as follows: Intel Core i5-4200M CPU @ 2.60GHz, 4.00 GB memory, AMDRadeonR7M265, 500GB hard disk, Windows 10. The Experimental Result is shown in Fig.2:

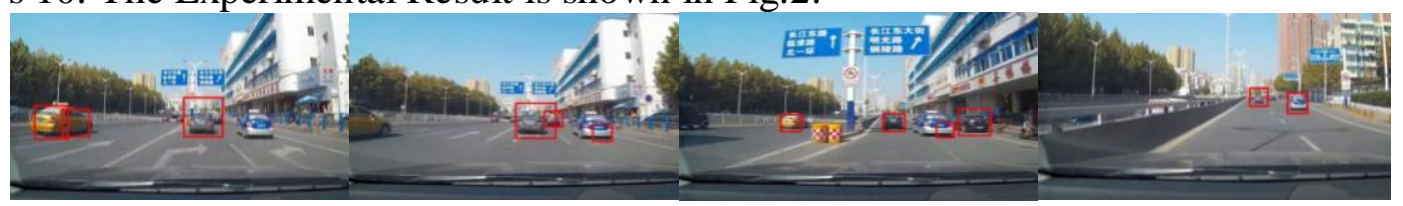

Fig.2 The result of identification

In the paper, the principle of monocular vision measurement based on feature points are used, after the camera has been calibrated, the images are processed, calculating the actual distance between the target and the camera. The comparison of the calculated data is shown in Table 2.

Table 2. The comparison of the calculated data

\begin{tabular}{|c|c|c|c|c|c|}
\hline Image ordinate $($ Pixel $)$ & 4 & 17 & 28 & 36 & 44 \\
\hline Actual distance $(m)$ & 7 & 8 & 9 & 10 & 11 \\
\hline Calculating distance $(m)$ & 7.2 & 8.1 & 9.0 & 9.9 & 11.1 \\
\hline
\end{tabular}

\section{Conclusion}

This paper analyzes the basic principle of vehicle identification based on machine vision. Through the camera's captured images preprocessing. It uses the Haar features of the samples to carry out sample training to train the classifier, to achieve the vehicle identification. Then it uses the principle of pinhole imaging to deduce the relationship between the image and the target distance. Finally, the vehicle distance and relative velocity in the process of running are calculated by the method of feature point measurement. By using the existing vehicle safety distance formula, we can carry out real-time monitoring of moving vehicle; it can carry on the early-warning to the vehicles which have the potential collision. From the data, the method is feasible and it has the minor error, which proves the effectiveness of the algorithm.

\section{Acknowledgments}

This study of the paper was supported by Natural Science Foundation of Jilin province (20170 101133JC), innovation and entrepreneurship training program for college students of Jilin University (2016B53483).

\section{References}

[1] Toroyan T. Global status report on road safety: time for action[J]. Injury Prevention, Vol.15(2009), No.4:p.286-286.

[2] Organization W H. Global status report on road safety - 2013: supporting a decade of action[J]. Injury Prevention, Vol.15(2013), No.4:p.286-286. 
[3] Zhu J, Zou H, Rosset S, et al. Multi-class AdaBoost[J]. Statistics \& Its Interface,Vol.2(2006), No.3:p.349-360.

[4]Viola P, Jones M. Fast and Robust Classification using Asymmetric AdaBoost and a Detector Cascade [J]. Advances in Neural Information Processing Systems, Vol.14(2002), :p.1311--1318.

[5]Yong X, Zhang L, Song Z, et al. Real-time vehicle detection based on Haar features and Pairwise Geometrical Histograms [C] IEEE International Conference on Information and Automation. IEEE, (2011):p.390-395.

[6] Lenser S, Veloso M. Visual sonar: fast obstacle avoidance using monocular vision[C]. IEEE International Conference on Intelligent Robots and Systems. IEEE, Voll.1(2003):p.886-891

[7]Chi C Y. Automatic safety driving distance control device for a vehicle: US, US5165497[P]. (1992). 